\title{
The peer review system of Archives of Plastic Surgery: Current status and plans for improvement
}

\section{Kyu Jin Chung}

Deputy Editor, Archives of Plastic Surgery

Department of Plastic and Reconstructive Surgery, Yeungnam University Hospital, Daegu, Korea

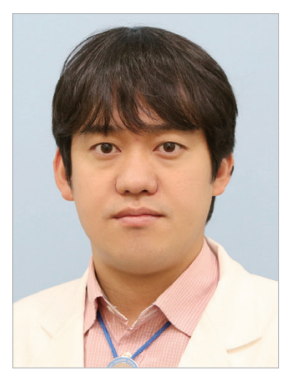

The role of peer reviewers for medical journals is to evaluate submissions for quality and reliability and to determine whether they deserve publication. The peer review system is considered a vitally important component of medical and scientific journal publishing, to the point that scientific journals have no choice but to depend on the peer review system in order to publish high-quality articles. When journals receive a heavy volume of submissions, they utilize the peer review system to select those with superior quality. Thus, to summarize, the peer review system can be defined as a process of selecting studies to be published in academic journals, improving their overall quality, and screening for any potential errors and weaknesses.

Despite the ubiquity and advantages of the peer review system, it is not perfect [1]. The evaluative process of peer review has not changed or been improved for several decades. A weakness of this system is that it is expensive and time-consuming. The evaluative process is inherently a laborious task that can take hours to days because it involves cross-referencing citations and reviewing the methodologies described in a submission. Furthermore, although peer reviewers are not compensated, the time invested in the review itself imposes opportunity costs.

The general belief that the peer review system is objective and reliable. However, it is inevitably vulnerable to criticisms and complaints from the authors of rejected studies who may regard the process as being inconsistent. If a reviewer is asked to evaluate his or her competitor's work, there is a possibility that the reviewer may deliberately delay or block the publication of the study, or even steal its original ideas. Despite these inherent weaknesses of the peer review system, many journals still utilize the process because no adequate alternative exists. Nonetheless, being a peer reviewer can provide easy access to the most recent studies and can be helpful for keeping one's knowledge up-todate.

Fake peer review is a phenomenon in which researchers take advantage of the peer review system, and in particular journals that allow authors to suggest peer reviewers, by entering email addresses for the suggested peer reviewers (either real scientists or invented personae) that the researchers themselves control. By impersonating peer reviewers, authors can dramatically increase the likelihood of their submission being accepted $[2,3]$. Such abuses call into question the legitimacy of the peer review process, and it is incumbent upon journal editors to work diligently to prevent such misconduct.

The content of a peer evaluation must be clear and concise in order to aid the editors in determining whether they should continue with the publication process of the submission. Accurately evaluating the methodology is an important part of the review process. Although it is important for medical and scientific studies to contain innovative and interesting content, inadequate descriptions of methodology suggest a vulnerability to bias and may cast doubt on the results of the research. Even if the submission is to be rejected, the content of the evaluation must be constructive so that the authors can improve the quality of their research and/or their presentation of the results.

Archives of Plastic Surgery (APS) has a total of 307 reviewers from 40 countries. As the official journal of the Korean Society 
for Plastic and Reconstructive Surgeons (KSPRS), APS implements strict vetting to select its reviewers in Korea from the pool of KSPRS members. Outside of Korea, there are 19 reviewers in the United States, 10 in Japan, 10 in Italy, and 10 in the United Kingdom. The special reviewers include two specialists in the field of statistics, one in parasitology, one in psychiatry, one in anesthesiology, and one in genetics. Fake peer review can be prevented by using qualified reviewers selected from the pool of board-certified plastic surgeons. Although APS does not yet have any need for author-recommended reviewers, as it already has over 300 professional reviewers available, such a need may arise in the future as more studies are submitted for evaluation. An analysis of APS evaluations between 2013 and 2018 indicated with an average evaluation period of 15.69 days and an average of 3.95 completed evaluations per reviewer.

Editors must play a central role throughout the process of peer review because they are responsible for selecting reviewers and making the final decision on whether to publish each study. In most cases, peer reviewers do not receive any formal training through written instructions or courses [4]. Although many journals provide reviewers with their own evaluative criteria, it is difficult for reviewers to learn a uniform set of evaluative skills, as these criteria differ across journals. As the official journal of KSPRS, APS offers annual lectures for peer reviewers through the KSPRS.

Many forms of peer review are available. Several methods are being introduced to improve the integrity of the peer review system [5], such as standardizing procedures, promoting procedural transparency, blinding reviewers from the authors' identities, reviewing protocols, training reviewers, promoting more rigorous selection and deselection of reviewers, utilizing electronic reviews, rewarding reviewers, providing detailed feedback to reviewers, using more checklists, and creating professional agencies. APS should apply these various improvement methods. To do so is necessary for better publication.

\section{NOTES}

\section{Conflict of interest}

No potential conflict of interest relevant to this article was reported.

\section{ORCID}

Kyu Jin Chung https://orcid.org/0000-0001-6335-1818

\section{REFERENCES}

1. Smith R. Peer review: a flawed process at the heart of science and journals. J R Soc Med 2006;99:178-82.

2. Ferguson C, Marcus A, Oransky I. Publishing: the peer-review scam. Nature 2017;515:480-2.

3. Qi X, Deng H, Guo X. Characteristics of retractions related to faked peer reviews: an overview. Postgrad Med J 2017;93: 499-503.

4. Benos DJ, Kirk KL, Hall JE. How to review a paper. Adv Physiol Educ 2003;27:47-52.

5. Dadkhah M, Kahani M, Borchardt G. A method for improving the integrity of peer review. Sci Eng Ethics 2018;24: 1603-10.

Correspondence: Kyu Jin Chung

Department of Plastic and Reconstructive Surgery, Yeungnam University Hospital, 170 Hyeonchungro, Nam-gu, Daegu 42415, Korea

Tel: +82-53-620-3480, Fax: +82-53-626-0705, E-mail: guzy7@hanmail.net

Received: 11 May 2019• Revised: 13 May 2019• Accepted: 13 May 2019 pISSN: 2234-6163 • elSSN: 2234-6171

https://doi.org/10.5999/aps.2019.00528 • Arch Plast Surg 2019;46:187-188 